\title{
An Online Optimal Explicit Guidance Scheme for Long Range Missiles with Solid Motors
}

\author{
Wael Mohsen Ahmed \\ School of Automation Science and Electrical Engineering \\ Beihang University \\ Beijing, China \\ waelsoliman@rocketmail.com
}

\begin{abstract}
By combining nonlinear model predictive static programming with dynamic inversion control, a hybrid robust explicit guidance scheme is developed for long range missiles with solid motors. The developed guidance technique can produce two successful guidance command profiles: a positive profile and a negative profile, where the negative profile solution provides relatively less control command magnitude at switch over time (i.e. time when the guidance is switched to dynamic inversion control) than guidance with the positive profile. However, the positive profile is more feasible for ballistic missile capability. The choice between the two profiles will depend on the constraints of the missile capability and mission requirement. The advantages of the developed guidance method are: 1) it is insensitive to energy uncertainties although the motor total impulse (i.e. the area under the thrust $\sim$ time curve) is not constant, 2) it can be implemented online due to its closed form nature, with less computational difficulties. The numerical simulations demonstrate the advantages above.
\end{abstract}

\section{INTRODUCTION}

Guidance of long range missiles with solid motors is our objective. In addition to the absence of thrust cutoff facility, the uncertainty in solid motor performance limits the knowledge of the actual burnout time and this may lead to the failure of the mission. To achieve the goal of intercepting, uncertainties should be considered in the guidance design. In this paper, we investigate a recently developed nonlinear optimal control design technique, named as Model Predictive Static Programming (MPSP) [11], in conjunction with the "dynamic inversion approach" [7] to develop an online robust explicit guidance scheme which is insensitive to energy uncertainties in the solid motors performance.

In this study, the mission of a two stage solid-solid vehicle has been considered. Starting point for the second stage was assumed to be a deterministic point. The task was to guide the vehicle in the boost phase during the second stage so that the missile falls on the required error basket at desired target point. As a simplified yet realistic assumption, the starting point of the second stage of the vehicle (also assumed to be the point of Guidance Loop Closure (GLC)) is considered to be well above the effective atmosphere [3]. In most of the cases, this is a realistic assumption. The earth model considered in the current work is non-rotating and spherical. Unlike a more realistic three dimensional trajectory [5], the considered mission is only planar. Furthermore, the vehicle is assumed to be a point mass [3].

\section{Dynamics and Mission Mathematical Model}

\section{A. Ex-Atmospheric Thrusted Flight}

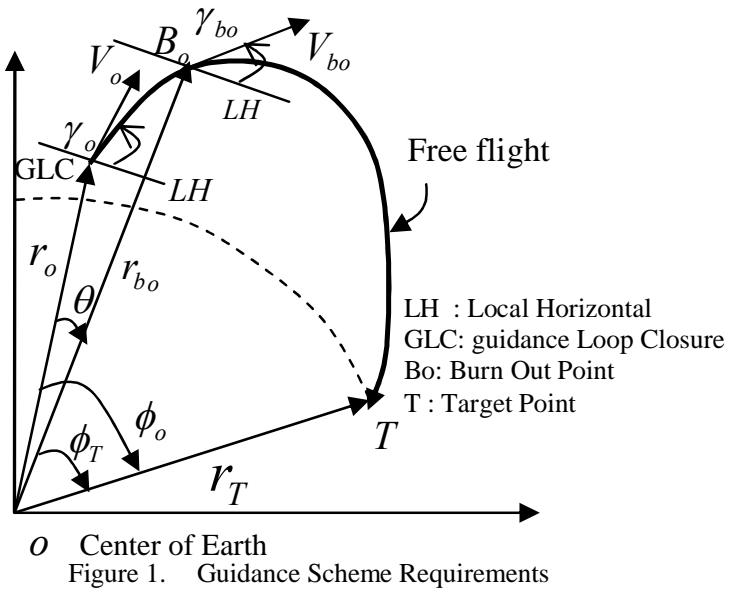

Above atmosphere, the vehicle experiences the forces only due to thrust and gravity. With the assumption that the earth is spherical and non-rotating, the point-mass dynamics of the vehicle beyond atmosphere is given by the following set of differential equations [3]

$$
\left[\begin{array}{c}
\dot{x}_{1} \\
\dot{x}_{2} \\
\dot{x}_{3} \\
\dot{x}_{4}
\end{array}\right]=\left[\begin{array}{c}
\dot{r} \\
\dot{V} \\
\dot{\gamma} \\
\dot{\phi}
\end{array}\right]=\left[\begin{array}{c}
V \sin \gamma \\
\frac{T}{m} \cos \delta-g \sin \gamma \\
-\left(\frac{T}{m V}\right) \sin \delta+\left(\frac{V}{r}-\frac{g}{V}\right) \cos \gamma \\
-\left(\frac{V \cos \gamma}{r}\right)
\end{array}\right]
$$


where, $\gamma$ is the flight path angle w.r.t the local horizontal, $T$ is the thrust level, $m$ is the mass of the vehicle, $V$ is the velocity of the vehicle, $g$ is the acceleration due to gravity, $\delta$ is shear angle (thrust deflection w.r.t velocity vector), and $\phi$ is the range angle to be covered.

After the thrusted phase and above effective atmosphere, the vehicle experiences only the gravitational field. In this condition, assuming a Spherical and Non-Rotating earth, adopting a polar co-ordinate system, after some analysis ([1], [2]), the free-flight closed form solution for the equation of motion for radial distance is given by:

$$
\frac{r_{b o}}{r}=\frac{1-\cos \theta}{\lambda \cos ^{2} \gamma_{b o}}+\frac{\cos \left(\theta+\gamma_{b o}\right)}{\cos \gamma_{b o}}
$$

where $\lambda=r_{b o} V_{b o}^{2} / G M, G M$ is the product of universal gravitational constant and mass of the earth, and $r(0)=r_{b o}$, $V(0)=V_{b o}, \quad \dot{r}(0)=V_{b o} \sin \gamma_{b o}$.

If it is desired to hit a target that is at distance $x$ along the surface of earth, then the angular distance to be covered is given by $\phi=x / \operatorname{Re}$ where $\mathrm{Re}$ is the radius of earth. In that case, $\theta=\phi$ and $r=r_{T}$, the radius for the target, the free flight equation in this case is known as the hit equation [2] which is given by

$$
\frac{r_{b o}}{r_{T}}=\frac{1-\cos \phi_{b o}}{\lambda \cos ^{2} \gamma_{b o}}+\frac{\cos \left(\phi_{b o}+\gamma_{b o}\right)}{\cos \gamma_{b o}}
$$

In equation (3), the subscript ' $b o$ ' in a variable denote the corresponding value of it at the burn out condition, i.e. at the beginning of the free flight. The free flight motion, governed by equation (3), is elliptic in nature if $\lambda<2$. If $\lambda \geq 2$, the vehicle reaches the escape velocity, and leaves the earth permanently. Moreover, for any target intercepting trajectory at a particular position $r_{b o}$ and velocity $V_{b o}$, the choice of $\gamma_{b o}$, is not unique, in which two choices of $\gamma_{b o}$ are possible and they are related to the range angle $\phi$ by $\gamma_{b o_{1}}+\gamma_{b o_{2}}=\frac{\pi}{2}+\frac{\phi}{2}$. In fact, one of these choices leads to a steep trajectory and the other leads to a shallow trajectory. Here we have aimed for a shallow trajectory (else, it is impossible to design an optimal explicit guidance scheme without a discontinuity in the shear angle history) [3].

\section{B. Guidance Design Using MPSP and Dynamic Inversion}

The objective of the guidance scheme is to compute the guidance command input to satisfy the hit equation at switch over time $\left(t_{s o}\right)$, in which the guidance algorithm is switched over to other guidance scheme based on dynamic inversion approach. In other words, the guidance scheme must make sure that the output $Y_{N}$ at final time goes to desired value $Y_{N}^{*}$ such that $Y_{N} \rightarrow Y_{N}^{*}$ at $t=t_{s o}$. For guidance with MPSP technique, one needs to start from a "guess history" of the control solution. In this section, we present a way to compute an error history of the control variable, which needs to be subtracted from the previous history to get an improved control history. This iteration continues until the objective is satisfied i.e. until $Y_{N} \rightarrow Y_{N}^{*}$ [12]. In this problem the state vector $X \hat{=}\left[\begin{array}{llll}r & V & \gamma & \phi\end{array}\right]^{T}$ and the control input $U \hat{=} \delta$ (guidance command). Using the Euler method, the discretized state dynamics is given by

$$
X_{K+1}=F\left(X_{K}, U_{K}\right)=X_{K}+\Delta t f\left(X_{k}, U_{K}\right)
$$

where $f\left(X_{K}, U_{K}\right)$ is right hand side expression of state equation in (1). From (3), the discretized output at $k=N$ i.e. $\left(t=t_{s o}\right)$ is given by

$$
y_{N}=r_{T}=\frac{r_{N}^{2} V_{N}^{2} \cos ^{2} \gamma_{N}}{G M\left(1-\cos \phi_{N}\right)+r_{N} V_{N}^{2} \cos \left(\phi_{N}+\gamma_{N}\right) \cos \gamma_{N}}
$$

Here $r_{T}$ is the radial distance from the center of the earth at target point. The guidance scheme should be robust against motor uncertainty. To achieve this objective, we expand the hit equation about the switch over time using Taylor series and neglecting higher order terms, we get

$$
y \cong y_{s o}+\dot{y}_{s o} \Delta t
$$

To make guidance scheme insensitive to actual burnout time, we force the sensitive term $\dot{y}_{s o}$ to be zero. If the height derivative term stays near zero, the height variation will be the minimum. Taking the advantage of this fact, we have chosen the output as $Y_{N}=\left[\begin{array}{ll}y & y_{s o}\end{array}\right]^{T}$. The error in outputs are calculated as follows

$$
d Y_{N}=Y_{N}-Y_{N}^{*}
$$

where $Y_{N}^{*}=\left[\begin{array}{ll}r_{T}^{*} & 0\end{array}\right]^{T}$ is desired output at target position, $r_{T}^{*}$ is aimed radial distance at the target. The aim here is to compute the guidance command sequence $\delta_{k}$, $k=1,2, \ldots,(N-1)$ so that $d Y_{N} \rightarrow 0$. To achieve this objective first we define the output error $d Y_{N}$ as

$$
d Y_{N}=\left[\frac{\partial Y_{N}}{\partial X_{N}}\right] d X_{N}
$$

From (4) the state error at time step $(k+1)$ is given by

$$
d X_{k+1}=\left[\frac{\partial F_{k}}{\partial X_{k}}\right] d X_{k}+\left[\frac{\partial F_{k}}{\partial U_{k}}\right] d U_{k}
$$

where $d X_{k}$ and $d U_{k}$ are the error of state and control at time step $k$ respectively. Expanding $d X_{N}$ as in (9) (for $k=N-1)$ and substituting it into (8), we get 


$$
d Y_{N}=\left[\frac{\partial Y_{N}}{\partial X_{N}}\right]\left(\left[\frac{\partial F_{N-1}}{\partial X_{N-1}}\right] d X_{N-1}+\left[\frac{\partial F_{N-1}}{\partial U_{N-1}}\right] d U_{N-1}\right)
$$

By expanding $d x_{k}, k=(N-2),(N-3), \ldots ., 1$ in a sequential manner arranging the terms, we get

$$
d Y_{N}=A d X_{1}+B_{1} d U_{1}+\ldots+B_{N-1} U_{N-1}
$$

Since the initial condition is specified, there is no error in the first term which means $d X_{1}=0$ with this (11) reduces to

$$
d Y_{N}=\sum_{k=1}^{N-1} B_{k} d U_{k}
$$

where

$$
B_{k} \stackrel{\Delta}{=}\left[\frac{\partial Y_{N}}{\partial X_{N}}\right]\left(\left[\frac{\partial F_{N-1}}{\partial X_{N-1}}\right] \ldots\left[\frac{\partial F_{k+1}}{\partial X_{k+1}}\right]\left[\frac{\partial F_{k}}{\partial U_{k}}\right]\right)
$$

Following the theory of static optimization the augmented cost function is given by

$$
\bar{J}=\frac{1}{2} \sum_{k=1}^{N-1} d U_{k}^{T} R_{k} d U_{k}+\lambda^{T}\left(d Y_{N}-\sum_{k=1}^{N-1} B_{k} d U_{k}\right)
$$

where $R_{k}>0$ (a positive definite matrix) is the weighting matrix. The necessary conditions of optimality are given by

$$
\begin{gathered}
\frac{\partial \bar{J}}{\partial d U_{k}}=R_{k} d U_{k}-B_{k}^{T} \lambda=0 \\
\frac{\partial \bar{J}}{\partial \lambda}=d Y_{N}-\sum_{k=1}^{N-1} B_{k} d U_{k}=0
\end{gathered}
$$

Solving for $d U_{k}$ from (14), we get

$$
d U_{k}=R_{k}^{-1} B_{k}^{T} \lambda
$$

Substituting $d U_{k}$ into (14), and assuming $A_{\lambda}$ to be nonsingluar, the solution of $\lambda$ is given by

$$
\lambda=A_{\lambda}^{-1} d Y_{N}
$$

where $A_{\lambda} \hat{=} \sum_{k=1}^{N-1} B_{k} R_{k}^{-1} B_{k}^{T}$. Substituting (17) into (16) yields

$$
d U_{k}=R_{k}^{-1} B_{k}^{T} A_{\lambda}^{-1} d Y_{N}
$$

Hence, the updated control at time step is given by

$$
U_{k}=U_{k}^{0}-d U_{k}=U_{k}^{0}-R_{k}^{-1} B_{k}^{T} A_{\lambda}^{-1} d Y_{N}
$$

It is clear from (19) that the updated control history solution is a closed form solution. Hence, this formulation is suitable for online implementation.

To explain the guidance design using dynamic inversion, first we define the height error at the target position as

$$
h_{T_{e}}=y-y^{*}
$$

where $y$ is same as $y_{N}$ in (5) and the tracking command is $h_{T_{e}}^{*}=\operatorname{Re}+65 \mathrm{~km}$. The goal here is to compute control histories $\delta$ such that

$$
\dot{h}_{T_{e}}+K h_{T_{e}}=0 \rightarrow \dot{y}+k\left(y-y^{*}\right)=0
$$

where $k>0$ is the chosen gain value.

$$
\dot{y}=\frac{\partial y}{\partial r} \dot{r}+\frac{\partial y}{\partial V} \dot{V}+\frac{\partial y}{\partial \gamma} \dot{\gamma}+\frac{\partial y}{\partial \phi} \dot{\phi}
$$

Substituting for $\dot{y}$ in (22) and after rearranging, we get

$$
\frac{\partial y}{\partial V} \dot{V}+\frac{\partial y}{\partial \gamma} \dot{\gamma}=\beta
$$

where

$$
\beta \hat{=}-\left(k\left(y-y^{*}\right)+\frac{\partial Y}{\partial \dot{r}} \dot{r}+\frac{\partial Y}{\partial \phi} \phi\right)
$$

substituting for $\dot{V}$ and $\dot{\gamma}$ in (23) from (1) and again we get

$$
\frac{\partial y}{\partial V} \frac{T}{m} \cos \delta+\frac{\partial y}{\partial \gamma}\left(-\frac{T}{m V}\right) \sin \delta=\beta+\beta_{a}
$$

where

$$
\beta_{a} \hat{=} \frac{\partial y}{\partial V} g \sin \gamma-\frac{\partial y}{\partial \gamma}\left(\frac{V}{r}-\frac{g}{V}\right) \cos \gamma
$$

To solve equation (25) we assume that

$$
a \cos \delta+b \sin \delta=c
$$

where $a=\frac{\partial y}{\partial V} \frac{T}{m}, b=\frac{\partial y}{\partial \gamma}\left(-\frac{T}{m V}\right)$, and $c=\beta+\beta_{a}$

We can reform (27) as

$$
\sqrt{a^{2}+b^{2}}\left(\frac{a}{\sqrt{a^{2}+b^{2}}} \cos \delta+\frac{b}{\sqrt{a^{2}+b^{2}}} \sin \delta\right)=c
$$

Now we can solve equation (29) by assume the following

$$
\sin \alpha=\frac{a}{\sqrt{a^{2}+b^{2}}}, \cos \alpha=\frac{b}{\sqrt{a^{2}+b^{2}}}, \quad \alpha=\tan ^{-1}\left(\frac{a}{b}\right)
$$

By substitute (30) into (29) we get

$$
\sin \alpha \cos \delta+\cos \alpha \sin \delta=\frac{c}{\sqrt{a^{2}+b^{2}}}
$$

Finally a closed form expression for guidance command to intersect the target is given by

$$
\delta=\sin ^{-1}\left(\frac{c}{\sqrt{a^{2}+b^{2}}}\right)-\alpha
$$




\section{Simulation Studies And Results}

\section{A. Data Generation and Assumptions}

For our simulation studies a two stage solid-solid motor has been considered. Starting point for the second stage is assumed to be a deterministic point [3]. The task is to guide the vehicle in the thrusted region of the second stage so that the missile falls within the required error bound at the desired target. The guidance is started at the guidance loop closure (GLC) time, which is assumed to be $1 \mathrm{sec}$ after the starting of the second stage. This was done so that sufficient amount of thrust is built up by that time for the guidance to be effective. From the results of the staging calculations [3], the first stage burn out velocity is fixed at $3300 \mathrm{~m} / \mathrm{s}$. Similarly the gross mass of the vehicle at the guidance loop closure is fixed at $13.1 \times 10^{3} \mathrm{~kg}$. The initial flight path angle is assumed to be $50^{\circ}$ (wrt. to the local horizontal). The range covered from the launch point is assumed to be $30 \mathrm{~km}$. The target for the boost guidance scheme is assumed to be at a height of $r_{T}^{*}=\mathrm{Re}+65 \mathrm{~km}$ simplified yet realistic assumption, the GLC point is assumed to be sufficiently above the effective atmosphere. The guidance cycle time is assumed to $100 \mathrm{msec}$. A height error of $\pm 1 \mathrm{~m}$ at the target is chosen to be the convergence criterion to terminate the MPSP algorithm.

\section{B. Numerical Results}

The numerical results demonstrate that dynamic inversion gain $\mathrm{k}$ must be chosen positive definite. In our assumption we choose the gain $k$ such that $0<k \leq 1$ in order to avoid the high harmonics associated with differential calculations. Moreover the developed guidance technique can produce two successful guidance command profiles: a positive profile and a negative profile. The choice between the two profiles will depend on the constraints of the missile capability and mission requirement.

\section{1) Handling Different Missions}

First simulation scenario is done by applying positive guidance command profile. Figure 2 shows the applied second stage nominal thrust time curve for solid motor. The numerical results of simulations are carried out for three different missions at the ranges of 2000, 3000 and $3500 \mathrm{~km}$. The successful converged trajectories and flight path angle $\gamma$ for different missions are shown in figures 3, 4. It can be seen from Figure 5 the predicted missile height errors $h_{t e}$ at target position, in which the height error $h_{t e}$ continuously goes close to zero value by MPSP guidance, and at certain time when $h_{t e}$ reaches $\pm 1 \mathrm{~m}$, the guidance instantaneously switches to dynamic inversion control technique, which has responsibility to keep $h_{t e}$ at zero value until final time $t_{f}$. If burnout occurs within this time period, the missile will intersect the target accurately. These results demonstrate that the developed guidance scheme can successfully be applied with the uncertainty in the burnout time. Figure 6 shows the developed guidance command history, while Figure 7 shows MPSP control response ( $d \delta$ ), which represents the updated control history solution. Figure 8,9 shows the dynamic inversion response represented by the closed loop feedback error, and dynamic inversion gain $k$ respectively.

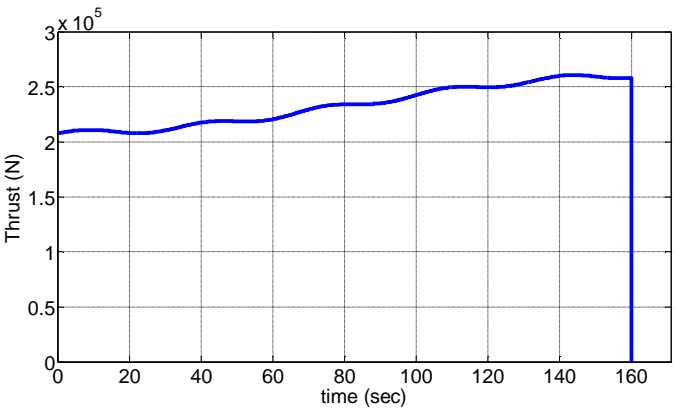

Figure 2. Thrust Time Curve

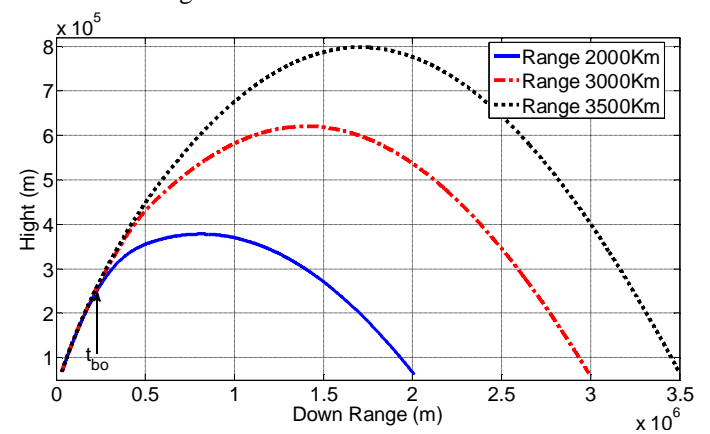

Figure 3. Converged Trajectories of Different Missions
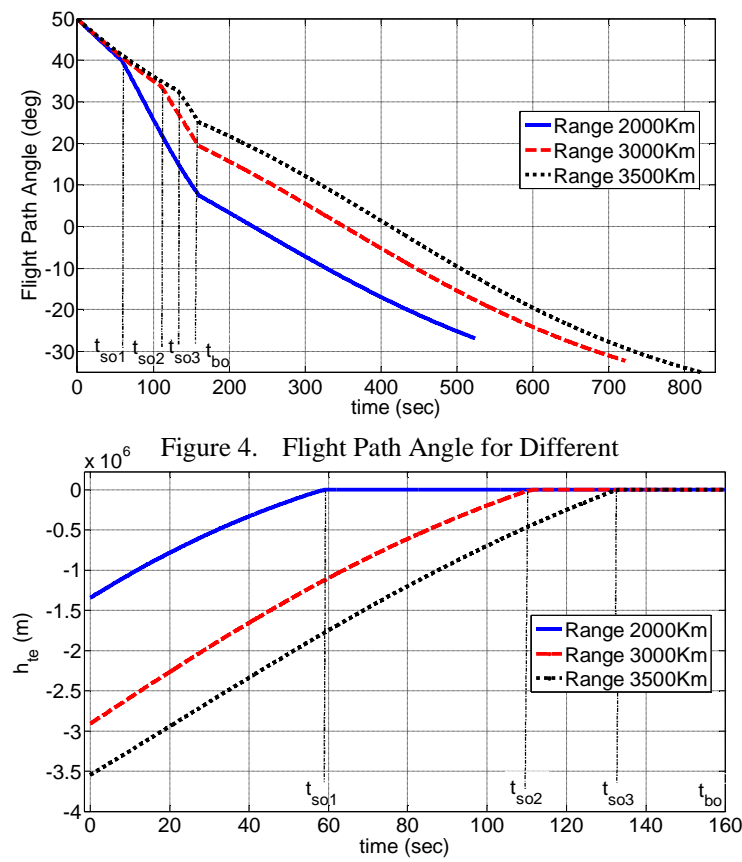

Figure 5. Predicted Height Error $\left(\mathrm{h}_{\mathrm{Te}}\right)$ 

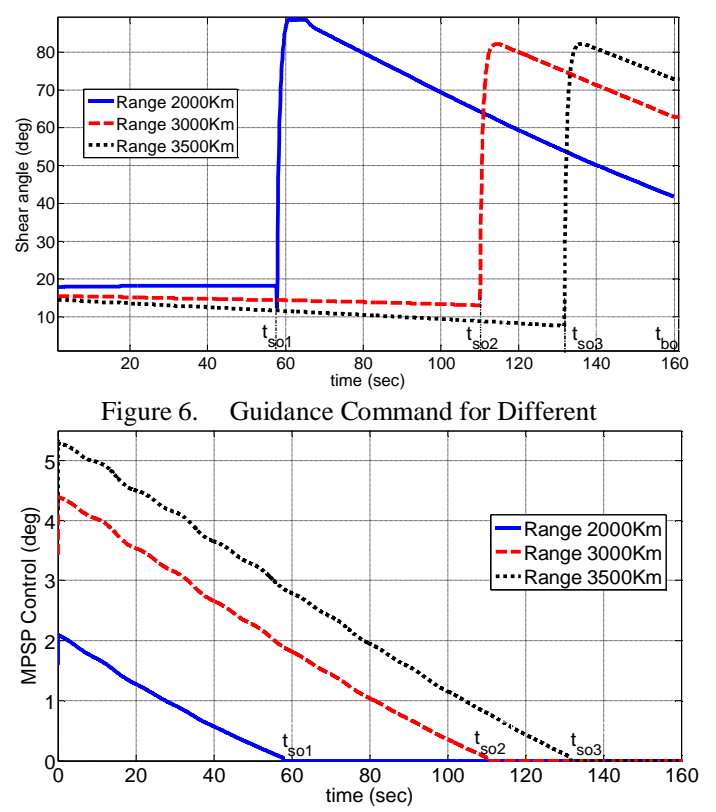

Figure 7. MPSP Control Response $d \delta$

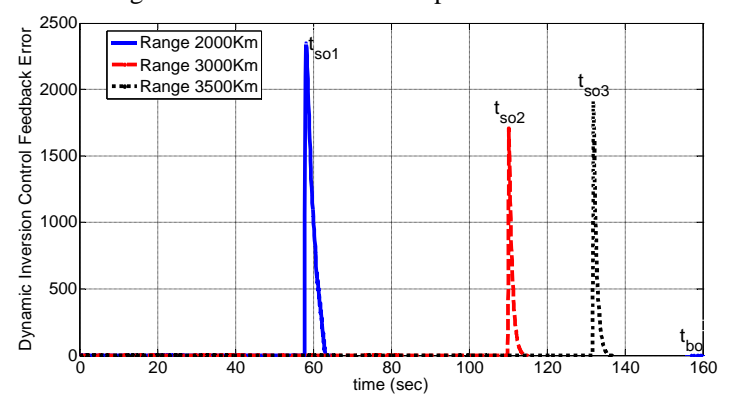

Figure 8. Dynamic Inversion Feedback Error

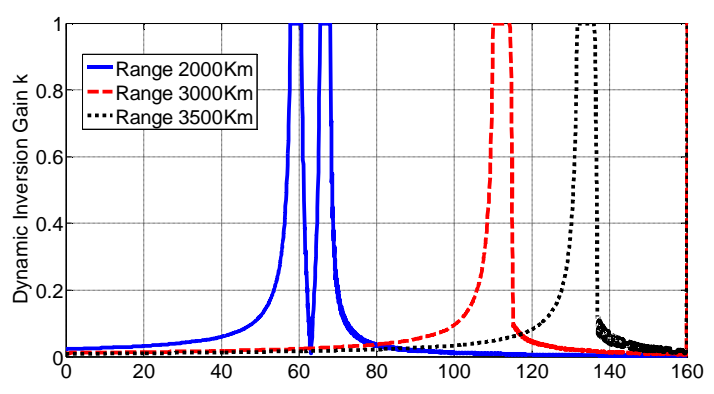

Figure 9. Dynamic Inversion Gain (k)

\begin{tabular}{|c|c|c|c|c|c|}
\hline $\begin{array}{c}\text { Range } \\
(\mathrm{Km})\end{array}$ & $\begin{array}{c}\text { Missile } \\
h_{\max }(\mathrm{Km})\end{array}$ & $\begin{array}{c}t_{s o} \\
(\mathrm{sec})\end{array}$ & $\begin{array}{c}t_{b o} \\
(\mathrm{sec})\end{array}$ & $t_{s o /} t_{b o}$ & $\begin{array}{c}\delta_{\max } \\
(\mathrm{deg})\end{array}$ \\
\hline 2000 & 3.7 & 58 & 160 & 0.36 & 87 \\
\hline 3000 & 6.2 & 110 & 160 & 0.68 & 82 \\
\hline 3500 & 7.9 & 131 & 160 & 0.81 & 82 \\
\hline
\end{tabular}

Table 1. First Scenario Results

It can be seen the gain $k$ is directly proportional to the loop feedback error. The core idea behind designing insensitive guidance to motor uncertainties relies on the technique of dynamic inversion, which continuously enforces $h_{T_{e}} \rightarrow 0$ and $\dot{h}_{T_{e}} \rightarrow 0$. We can see at switch over time $t_{s o}$ the gain $k$ gets its peak value, which forces the guidance command to get its highest magnitude. The results are tabulated in Table 1.

Second simulation scenario is done by choosing the negative guidance profile.

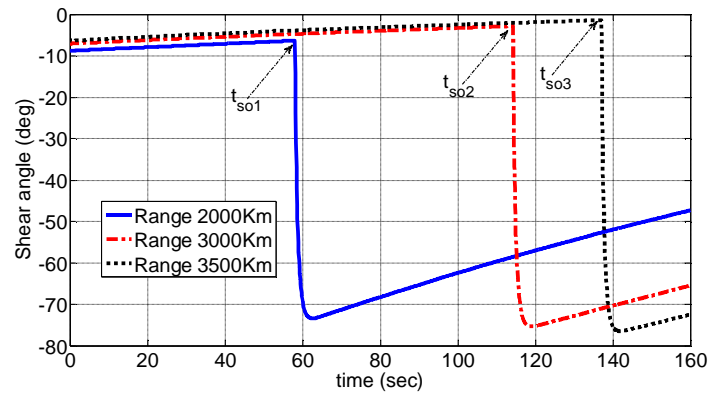

Figure 10. Guidance Command for Different Missions

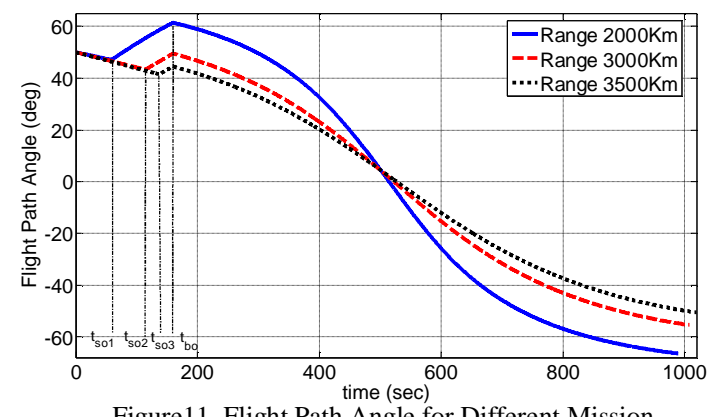

Figure11. Flight Path Angle for Different Mission

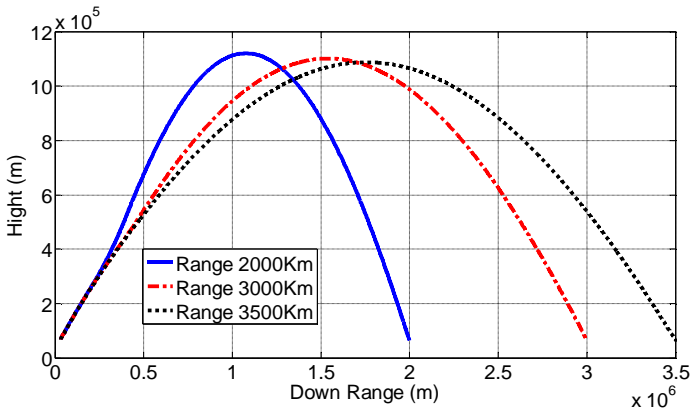

Figure 12. Converged Trajectories

\begin{tabular}{|c|c|c|c|c|c|}
\hline $\begin{array}{c}\text { Range } \\
(\mathrm{Km})\end{array}$ & $\begin{array}{c}\text { Missile } \\
h_{\max }(\mathrm{Km})\end{array}$ & $\begin{array}{c}t_{s o} \\
(\mathrm{sec})\end{array}$ & $\begin{array}{c}t_{b o} \\
(\mathrm{sec})\end{array}$ & $t_{\text {so/ }} t_{b o}$ & $\begin{array}{c}\left|\delta_{\max }\right| \\
(\mathrm{deg})\end{array}$ \\
\hline 2000 & 11 & 58 & 160 & 0.36 & 73 \\
\hline 3000 & 10.8 & 114 & 160 & 0.71 & 75 \\
\hline 3500 & 10.5 & 137 & 160 & 0.85 & 76 \\
\hline
\end{tabular}

Table2. Second Scenario Results

The missile succeeded to intercept the target, with higher converged trajectories. Moreover the developed guidance 
command history shown in Figure 10 has less control command magnitude at switch over time w.r.t positive profile. The associated flight path angle and converged trajectory are shown in figures 11,12 respectively. The results of second scenario tabulated in Table 2.

\section{2) Handling the Motor Uncertainties}

It has already been pointed out, the Thrust-time $(\mathrm{T} \sim \mathrm{t})$ curve of any Solid Motor can never be predicted exactly. In this section the simulation scenarios are done by applying different motor performances with un-constant total impulse in other word the area under the $\mathrm{T} \sim \mathrm{t}$ curve is not constant. From these specifications we assumed the sold motor performances as shown in Figure 13. The successful missions for same range applying different solid motor performances can be seen in figures 14, 15, 16.

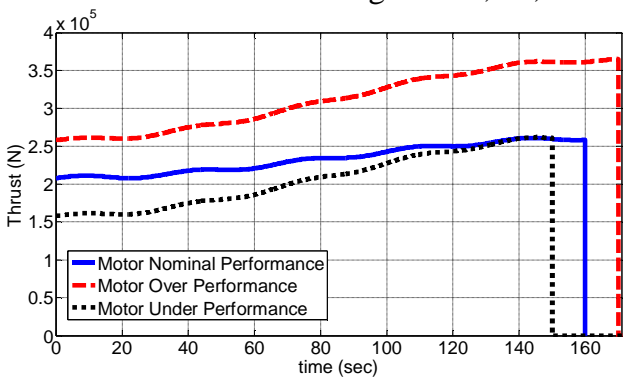

Figure 13. Thrust Time Curve with Different Performances
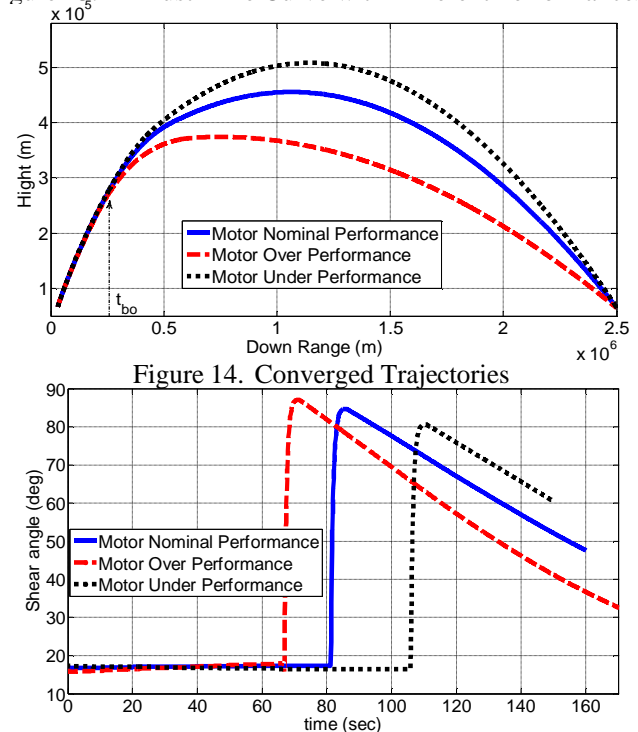

Figure 15. Solution of $\delta$ with different Motor Performances

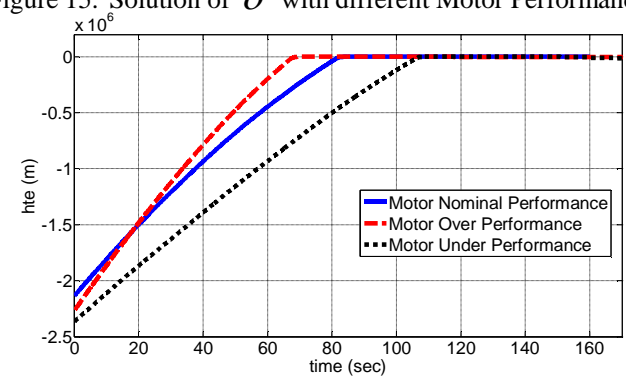

Figure 16. Predicted Height Error at Target
We can see the switch over time $t_{s o}$ is affected by the variations in the motor performances, in which the motor with over performance (higher thrust) lead to earlier $t_{s o}$ than the motor with under performance, but still the developed guidance able to steer the missile to intercept the target under only one condition, that total impulse must be enough to cover the required range.

\section{CONCLUSIONS}

By combining nonlinear MPSP with dynamic inversion control, a hybrid robust explicit guidance scheme is developed for long range missiles with solid motors.

The developed guidance technique can produce two successful guidance command profiles: a positive profile and a negative profile, where the negative profile solution provides relatively less control command magnitude at switch over time (i.e. time when the guidance is switched to dynamic inversion control) than guidance with the positive profile. However, the positive profile is more realistic for ballistic missile capability. The choice between the two profiles will depend on the constraints of the missile capability and mission requirement.

In addition the results demonstrate that dynamic inversion gain $k$ must be chosen positive definite. The limited gain $k$ is chosen such that $0<k \leq 1$ in order to avoid undesired peaks value associated with differential calculation. Moreover, the switch over time can be indicated by the peak value of dynamic inversion gain $k$.

\section{REFERENCES}

[1] Battin, R. H. (1982). Guidance Evolution - A Personal Narrative. Journal of Guidance, Control and Dynamics. Vol. 5. pp. 97-110.

[2] Zarchan, P (1997). Tactical and Strategic Missile Guidance. Proceeding in Astronautics and Aeronautics, AIAA Tactical Missile Series. Vol. 176. pp. 97-110.

[3] Padhi, R. (1999). An Optimal Explicit Guidance Scheme for Ballistic Missile with Solid Motors. Proceedings of AIAA Guidance, Navigation, and Control Conference. AIAA-4140.

[4] Bryson, A. E. and Y. C. Ho (1975). Applied Optimal Control. Hemisphere Publishing Corporation.

[5] Padhi, R., M. Kothari and A. Kamath. Model Predictive Static Programming: A Promising Real-time Technique for Optimal Control Design. Technical Report: IISc/AE/LINCGODS/RP/2006/01.

[6] Greensite, A. L. (1970) Analysis and Design of Space Vehicle Flight Control System. Spartan Bok.

[7] Enns, D., D. Hendrick, R. Hendrick and G. Stein (1994). Dynamic Inversion: An Evolving Methodology for Flight Control Design. International Journal of Control, Vol. 59, No. 1, pp.71-91.

[8] Slotine, J. E and W. Li. Applied Nonlinear Control. PrenticeHall.

[9] Khalil, H. K. Nonlinear System. Prentice Hall Inc., New Jersey, USA.

[10] Padhi, R. and M. Kothari (2005). A Closed Form Nonlinear Explicit Guidance Scheme for Long Range Flight Vehicles.

[11] P. N. Dwivedi, A.Bhattacharyya , and Radhakant Padhi (2008). Computationally Efficient Suboptimal Mid Course Guidance Using Model Predictive Static Programming (MPSP). The International Federation of Automatic Control Seoul, Korea, July 6-11, 2008.

[12] M. Kothari, and Padhi, R. Energy-insensitive Guidance of Solid Motor Propelled Long Range Flight Vehicles. The International Federation of Automatic Control Seoul, Korea, July 6-11, 2008. 